\title{
Fast post-adiabatic waveforms in the time domain: Applications to compact binary coalescences in LIGO and Virgo
}

\author{
Deyan P. Mihaylov $\odot,{ }^{1, *}$ Serguei Ossokine, ${ }^{1}$ Alessandra Buonanno, ${ }^{1,2}$ and Abhirup Ghosh $\odot^{1}$ \\ ${ }^{1}$ Max Planck Institute for Gravitational Physics (Albert Einstein Institute), \\ Am Mühlenberg 1, Potsdam 14476, Germany \\ ${ }^{2}$ Department of Physics, University of Maryland, College Park, Maryland 20742, USA
}

(Received 14 May 2021; accepted 25 October 2021; published 29 December 2021)

\begin{abstract}
We present a computationally efficient (time-domain) multipolar waveform model for quasicircular spinaligned compact binary coalescences. The model combines the advantages of the numerical-relativity informed, effective-one-body (EOB) family of models with a post-adiabatic solution of the equations of motion for the inspiral part of the two-body dynamics. We benchmark this model against other state-of-theart waveforms in terms of efficiency and accuracy. We find a speed-up of one to two orders of magnitude compared to the underlying time-domain ЕОВ model for the total mass range 2-100 $M_{\odot}$. More specifically, for a low total-mass system, such as a binary neutron star with equal masses of $1.4 M_{\odot}$, like GW170817, the computational speedup is around 100 times; for an event with total mass $\sim 40 M_{\odot}$ and mass ratio $\sim 3$, like GW190412, the speedup is by a factor of $\sim 20$, while for a binary system of comparable masses and total mass of $\sim 70 M_{\odot}$, like GW150914, it is by a factor of $\sim 10$. We demonstrate that the new model is extremely faithful to the underlying ЕОВ model with unfaithfulness less than $0.01 \%$ across the entire applicable region of parameter space. Finally, we present successful applications of this new waveform model to parameter estimation studies and tests of general relativity.
\end{abstract}

DOI: 10.1103/PhysRevD.104.124087

\section{INTRODUCTION}

Since 2015, the detections of gravitational waves (GW s) have yielded a wealth of remarkable discoveries [1-8]. In the three observing runs [7,8] of the Advanced LIGO [9] and Advanced Virgo [10] detectors, a total of 50 events have been observed and confirmed; among these are both binary black hole $(\mathrm{BBH})$ mergers and binary neutron star (BNS) mergers [11]. Particularly interesting are the discoveries of binaries GW190412 with mass ratio 3 [12] and GW190814 (which could be the first ever detected merger of a black hole and a neutron star) [13] with mass ratio 10. GW190521 is the most massive binary detected so far with a total mass of $150 M_{\odot}$ [14].

Detections of compact binary mergers are expected to increase in the coming years $[15,16]$ : during the upcoming LIGO and Virgo observing runs [17], and with future ground-based detectors like the Einstein Telescope [18] and Cosmic Explorer [19], and the space-based mission LISA

\footnotetext{
*deyan@aei.mpg.de

Published by the American Physical Society under the terms of the Creative Commons Attribution 4.0 International license. Further distribution of this work must maintain attribution to the author(s) and the published article's title, journal citation, and DOI. Open access publication funded by the Max Planck Society.
}

[20]. Extracting information from such GW detections relies on accurate and computationally efficient models of the gravitational waveforms, which are emitted during coalescence $[21,22]$. Firstly, the estimation of the binary parameters of a typical event (using Bayesian inference, Markov chains, or similar methods) requires on the order of several million evaluations of the waveform models $[23,24]$. On the other hand, the GW phase needs to be accurate to less than a cycle of the binary in order to avoid ambiguity in the estimations $[25,26]$. Upcoming runs, as well as future detectors, will require even better waveform accuracy in order to reliably identify and analyze GW events [27]. Accurately identifying the properties of a large population of binaries will allow us to make inferences on scenarios of compact-object binary formation [28], and also carry out more stringent tests of general relativity (GR) in the highly dynamical, strong-field regime [29]. For these reasons, work on more advanced and innovative waveform models continues for LIGO, Virgo, and future GW missions.

Gravitational-wave models that include the inspiral, merger, and ringdown stages of a compact binary coalescence have been developed using the effective-one-body (EOB) formalism [30-36] (notably the SEOBNR [37-46] and TEOBResums [47-50] waveform models), and the inspiral-merger-ringdown phenomenological approach [51-59] (i.e., the IMRPhenom models). The EOB families 
of models employ a semianalytic approach that combines an analytical description of the spinning two-body dynamics and gravitational radiation for the entire coalescence with numerical relativity (NR) information in the strongfield merger-ringdown regime [60-68]. Here, we will focus on the SEOBNR waveform models, which have been extensively used by LIGO and Virgo detectors to observe $\mathrm{GW}$ signals and infer astrophysical properties (e.g., see Refs. [7,8]) and test GR (e.g., see Ref. [29]).

The time-domain SEOBNR models $[43,45,46]$ are routinely employed in data analysis for sufficiently high-mass binaries with the LIGO Algorithm Library (LAL) Inference codes [24], while, for generic-mass binaries, fast parameter-estimation codes are required [69]. Nevertheless, the time for generating a waveform in the low-mass regime $\left(\lesssim 5 M_{\odot}\right)$ can be on the order of $\sim 100 \mathrm{~s}$ or even longer starting at $20 \mathrm{~Hz}$. Thus, there are parts of the binary's parameter space, for which the time-domain SEOBNR models are not suitable for direct use in parameter estimations without further optimizations, like the ones discussed in the current publication, which was originally introduced in Refs. [70,71]. Alternative methods have been developed in order to afford speedy data analysis for GW s. Reduced order modeling and surrogate techniques [72-81] have been successfully applied to EOB waveform models $[43,72,73,75,79]$ and to pure NR-waveforms $[74,76,78,82,83]$. These methods work by decomposing and interpolating the waveforms on a sparse grid in time or frequency domain, and then using interpolation or more sophisticated statistical methods to obtain the fitting parameters across the binary's parameter space under study. The resulting waveform model is then verified for accuracy against an independent testing set. However, although very successful, such models suffer from certain limitations. By construction, they are restricted to confined regions of the parameter space, and have to be developed from scratch if the underlying time-domain model is updated-for example when more physical effects are included or higher-order post-Newtonian parameters are added to make these waveforms more accurate.

Here, we develop the multipolar SEOBNRv4HM_PA waveform model for spin-aligned compact binaries moving on quasi-circular orbits. This model is a computationally cheaper version of the time-domain SEOBNRv4HM waveform model [45] and, as such, it includes higher-order harmonics (or higher modes, HM), which are important for asymmetric mass-ratio binaries, high-mass and highinclination systems [84-89]. In the SEOBNRV4HM_PA waveform model, the binary dynamics is solved using a post-adiabatic (PA) approach. The latter was proposed and applied to the TEOBResums model in Refs. [70,71,90,91] and used in all subsequent publications (see, e.g., Ref. [92-95] and references therein). It was also implemented in LALSimulation. In the PA method, the inspiral evolution (until the last few orbits before merger) is approximated by an adiabatic solution of the (ordinary differential) equations of motion, with post-adiabatic corrections added iteratively up to the order needed to achieve the desired accuracy. In this work we apply this technique to construct a fast and accurate aligned-spin dynamics based on the SEOBNRV4 model [43] and implement it in LALSimulation. The speed-up and accuracy benchmarks are supported by applications of the PA waveform model for parameter estimation studies and tests of GR.

The paper is organized as follows. Section II of this article reviews the EOB dynamics in the PA approximation for arbitrary Hamiltonians. Section III presents the implementation of this method in the LALSimulation waveform model library (as approximant SEOBNRV4HM_PA), and benchmarks the model against other established EOB models. Section IV presents two parameter estimation (PE) studies using the SEOBNRV4HM_PA model. In Sec. V the model is applied to a ringdown test of GR. Section VI concludes the article with a discussion of the significance of this work and its possible future directions. We shall work in natural units $G=1=c$.

\section{POST-ADIABATIC APPROXIMATION TO THE INSPIRAL DYNAMICS}

The EOB formalism provides an analytical description of the GW emission from the process of binary coalescence, including inspiral, merger, and ringdown [30,31]. The accuracy of this description can be further improved by calibrating against NR simulations.

A binary system composed of two BH s moving on a quasicircular orbit with spins aligned or antialigned (henceforth, spin-aligned for short) with the orbital angular momentum is described by four parameters: the component masses $m_{1}$ and $m_{2}$, and the (dimensionless) spins $\chi_{1}=$ $S_{1} / m_{1}^{2}$ and $\chi_{2}=S_{2} / m_{2}^{2}$. In the EOB approach the (centerof-mass) two-body dynamics is mapped onto the dynamics of an effective body of mass $\mu=m_{1} m_{2} /\left(m_{1}+m_{2}\right)$, which moves in a deformed Kerr spacetime of mass $M=m_{1}+m_{2}$, the deformation parameter being the symmetric mass ratio $\nu=\mu / M$. The conservative two-body dynamics is obtained from the EOB Hamiltonian [30,39]:

$$
H=M \sqrt{1+2 \nu\left(\frac{H_{\mathrm{eff}}}{\mu}-1\right)}-M,
$$

where $H_{\text {eff }}$ is the Hamiltonian that describe the motion of the effective body of mass $\mu$ and spin $S_{*}=\left[\left(m_{2} / m_{1}\right) S_{1}+\right.$ $\left.\left(m_{1} / m_{2}\right) S_{2}\right] / M^{2}$ in the (deformed) Kerr spacetime of mass $M$ with spin $S=S_{1}+S_{2}$.

For aligned-spin binaries, the motion is constrained to a fixed plane. Thus, we use polar coordinates and introduce the phase-space dimensionless variables 
$\left(r, \varphi, p_{r_{*}}, p_{\varphi}\right)$ related to the physical ones through the following expressions

$$
r=\frac{R}{M}, \quad p_{r_{*}}=\frac{P_{R_{*}}}{\mu}, \quad p_{\varphi}=\frac{P_{\varphi}}{\mu M} .
$$

The radial momentum $p_{r_{*}}$ is conjugate to the tortoise coordinate of the deformed spacetime $r_{*}[38,96]$. The dissipative effects in the EOB formalism are described by the radiation-reaction force [31,35-37]

$$
\mathcal{F}=\frac{\Omega}{16 \pi} \frac{\boldsymbol{p}}{|\boldsymbol{L}|} \sum_{\ell=2} \sum_{m=-\ell}^{\ell} m^{2}\left|d_{L} h_{\ell m}\right|^{2}
$$

where $\Omega$ is the angular orbital frequency, $\boldsymbol{L}$ is the orbital angular momentum, $d_{L}$ is the luminosity distance and $h_{\ell m}$ are the gravitational modes far from the source. In this setup, the equations of motion read [38]:

$$
\begin{aligned}
\frac{\mathrm{d} r}{\mathrm{~d} t} & =\frac{\mathrm{d} p_{r_{*}}}{\mathrm{~d} p_{r}} \frac{\partial H}{\partial p_{r_{*}}}, \\
\frac{\mathrm{d} \varphi}{\mathrm{d} t} & =\frac{\partial H}{\partial p_{\varphi}}, \\
\frac{\mathrm{d} p_{r_{*}}}{\mathrm{~d} t} & =-\frac{\mathrm{d} p_{r_{*}}}{\mathrm{~d} p_{r}} \frac{\partial H}{\partial r}+\mathcal{F}_{r}, \\
\frac{\mathrm{d} p_{\varphi}}{\mathrm{d} t} & =\mathcal{F}_{\varphi} .
\end{aligned}
$$

Here, $t=T / M$ is a dimensionless time variable.

The usual procedure employed in EOB waveform models involves solving the Eqs. (2.4) numerically, using an ordinary differential equation (ODE) integrator with a suitable time step and initial conditions. This is often computationally expensive (especially for longer waveforms), and is one of the bottlenecks for efficiently generating the EOB waveform. The post-adiabatic (PA) approximation $[31,70,90]$ converts the ODE equations of motion into a set of nonlinear algebraic equations which need to be solved numerically, but have a lower computational cost associated with them.

The adiabatic approximation assumes that the dynamics is comprised of a sequence of circular orbits. As such, there is no radiation reaction, hence $\mathcal{F}_{\varphi}=0$ and $p_{r_{*}}$ vanishes. Hence, the leading-order orbital angular momentum $p_{\varphi}$ can be calculated at a given radius from Eq. (2.4c):

$$
\left.\frac{\partial H}{\partial r}\right|_{p_{r_{*}}=0, p_{\varphi}, r}=0 .
$$

The post-adiabatic approximation assumes that the radiation reaction $\mathcal{F}_{\varphi}$ which can be used to furnish $p_{r_{*}}$ through a combination of Eqs. (2.4a) and (2.4c):

$$
\frac{\mathrm{d} p_{\varphi}}{\mathrm{d} r} \frac{\partial H}{\partial p_{r_{*}}}-\mathcal{F}_{\varphi}=0
$$

At the post-post-adiabatic level, one can use the newly obtained approximation for $p_{r_{*}}$ to additionally correct the orbital angular momentum $p_{\varphi}$, this time utilising Eqs. (2.4b) and $(2.4 \mathrm{~d})$ :

$$
\frac{\partial H}{\partial p_{r}}+\frac{\partial H}{\partial r} \frac{\mathrm{d} r}{\mathrm{~d} p_{r_{*}}}-\frac{p_{r_{*}}}{p_{\varphi}} \mathcal{F}_{\varphi}=0 .
$$

This approximation procedure can be iterated further, and the procedure for obtaining the corrections to the leading-order solution $\left(p_{r_{*}}, p_{\varphi}\right)=\left(0, j_{0}(r)\right)$ can be formalized in the following way, as described in [70]. For each value of the radial coordinate $r$, the radiation reaction can be written as an expansion in a formal parameter $\epsilon$

$$
\mathcal{F}_{\varphi}(r)=\sum_{n=0}^{\infty} \epsilon^{2 n+1} \mathcal{F}_{2 n+1}(r)
$$

Therefore, the solutions of the EOB equations of motion can also be written as an expansion in powers of this fictitious parameter:

$$
\begin{aligned}
& p_{\varphi}(r)=j_{0}(r)\left[1+\sum_{n=1}^{\infty} \epsilon^{2 n} \phi_{2 n}(r)\right]^{1 / 2}, \\
& p_{r_{*}}(r)=\sum_{n=0}^{\infty} \epsilon^{2 n+1} \rho_{2 n+1}(r) .
\end{aligned}
$$

The PA procedure allows for the two momenta to be calculated with arbitrary precision by adding more terms in the expansions above. The corrections at $(n, n+1)$ th PA order can be found by iteratively solving Eq. (2.6) for $p_{r_{*}}$ and Eq. (2.7) for $p_{\varphi}$. In solving these two equations, one must remember that all other variables (apart from the unknown one) must be kept at their most recent PA order. This procedure can be repeated as many times as necessary, until the desired accuracy in terms of powers of $\epsilon$ is achieved [70].

In practice, we proceed as follows. As a start, a radial grid is constructed for the part of the two-body dynamics where the PA approximation is to be applied, between two radii $r_{\max }$ and $r_{\min }$. At each node in this grid, the adiabatic solution $j_{0}(r)$ is obtained through Eq. (2.5) - it serves as the leading-order uncorrected values for the orbital angular momentum (the uncorrected value for $p_{r_{*}}$ is chosen as 0 everywhere on the grid). To obtain the $N$ th order PA approximation, the momenta $p_{r_{*}}(r)$ and $p_{\varphi}(r)$ are computed through Eqs. (2.6) and (2.7), respectively, at each point in the grid and this part is repeated up to the chosen PA order $N$. Whenever radial derivatives of the corrected quantities need to be computed [for instance, $\mathrm{d} p_{\varphi} / \mathrm{d} r$ in 
Eq. (2.6)], this is performed numerically on the grid. Finally, the time $t$ and the orbital phase $\varphi$ are obtained through numerical integration

$$
\begin{aligned}
t(r) & =\int_{r_{\min }}^{r_{\max }} \mathrm{d} r\left(\frac{\partial H}{\partial p_{r_{*}}}\right)^{-1}, \\
\varphi(r) & =\int_{r_{\min }}^{r_{\max }} \mathrm{d} r\left(\frac{\partial H}{\partial p_{\varphi}}\right)\left(\frac{\partial H}{\partial p_{r_{*}}}\right)^{-1} .
\end{aligned}
$$

The waveform is built from the PA dynamics using the same prescription as the standard EOB waveform model. The waveform strain $h(t)=h_{+}(t)-i h_{\times}(t)$ can be decomposed into multipoles according to

$$
h(t)=\frac{1}{d_{L}} \sum_{\ell=2}^{\ell_{\max }} \sum_{m=-\ell}^{\ell} h_{\ell m}(t)_{-2} Y_{\ell m}(\theta, \phi),
$$

where $d_{L}$ is the distance from the detector to the source, and ${ }_{-2} Y_{\ell m}(\theta, \phi)$ are the spin-weighted spherical harmonics for $s=-2 . \ell_{\max }$ is the highest-order multipole which is calculated. More detailed accounts of the procedure for generating EOB waveforms can be found in Ref. [42,47,48]. A robust implementation of the PA dynamics for an arbitrary spin-aligned EOB Hamiltonian is presented in the Sec. III.

\section{IMPLEMENTATION IN LIGO ALGORITHM LIBRARY}

We have implemented the post-adiabatic (PA) inspiral dynamics model described in Sec. II in LALSuite [97] and it is available through the SEOBNRV4HM_PA waveform model approximant.

When this model is used, the dynamics of the binary system, starting from the initial separation $r_{0}=r_{\max }$ until some final separation $r_{\min }$ is approximated with the PA procedure described in Sec. II. The radius at which the PA procedure is terminated, $r_{\min }$, as well as the size of the grid $\mathrm{d} r$, are empirically chosen to ensure that the faithfulness of the waveform is maximized while keeping the computational cost minimal. Around $10^{4}$ waveforms were generated, covering the space of binary parameters and exploring the effects of varying these two parameters. In each case we compute the unfaithfulness and choose values for these parameters that ensure the fastest waveform generation while still being sufficiently accurate. We find that the following prescription satisfies these requirements:

$$
r_{\min }=1.6 r_{\text {ISCO }} \quad \text { and } \quad \mathrm{d} r=0.3 .
$$

Furthermore, the PA order is a free parameter of our model, with the default being 8th order. Our studies show that lower orders cannot always achieve the desired accuracy, while higher orders incur computational cost without further improving the solution, or can be prone to numerical noise (e.g., above 12th order).

The PA approach is independent of any particular form of the Hamiltonian, and here we focus on the SEOBNRv4HM Hamiltonian $[39,43] .{ }^{1}$ Our procedure is set up to use either analytical or numerical derivatives of the EOB Hamiltonian (e.g., $\partial H / \partial r$ ), this giving additional flexibility to the user. The numerical derivatives in Eqs. (2.5), (2.6), and (2.7) are computed using an 8th-order finite difference method [98-100], while numerical integration is performed using a standard cube-spline quadrature algorithm $[101,102]$.

In order to provide an implementation of the waveform model that is maximally efficient while preserving the faithfulness at each point in parameter space, a number of further changes are introduced to the algorithms for calculating the binary dynamics and for computing the waveform modes. These changes are summarized below:

(1) Analytic derivatives are used both during the PA routine and the final ODE integration (where the approximant SEOBNRV4_opt is used $[43,103,104])$. Analytic derivatives are computationally more efficient than finite-difference methods, and therefore provide valuable speedup for computing the binary dynamics.

(2) A larger integration step is used for the final part of the dynamics calculation (using the SEOBNRv4_opt model), which speeds up the ODE integration significantly.

(3) Following Refs. [43,103], quantities which do not vary with the mode numbers $(\ell, m)$ are precomputed instead of being repeatedly generated during each iteration. This helps to remove a large portion of the computational overhead in building the waveform modes.

(4) Finally, the waveform is computed over a nonuniformly-spaced time grid, which is comprised of the sparse grid for PA approximation, and the denser grid for the final part of the dynamics (plunge and merger). This speeds up the waveform generation considerably as waveform generation on an equally spaced grid is expensive. To obtain the final modes on an equally spaced grid we follow the interpolation approach described in [81].

\section{A. Computational performance of the model}

The SEOBNRV4HM_PA model was benchmarked against other relevant waveform models which are

\footnotetext{
${ }^{1}$ We note that in Ref. [71] the authors derived the equations of motion of the (uncalibrated) Hamiltonian used in the SEOBNRV4HM model. However, they employed a form of the Hamiltonian that differs from the one used in the SEOBNRV4HM waveform model $[43,45]$. Thus, we could not take advantage of their findings.
} 


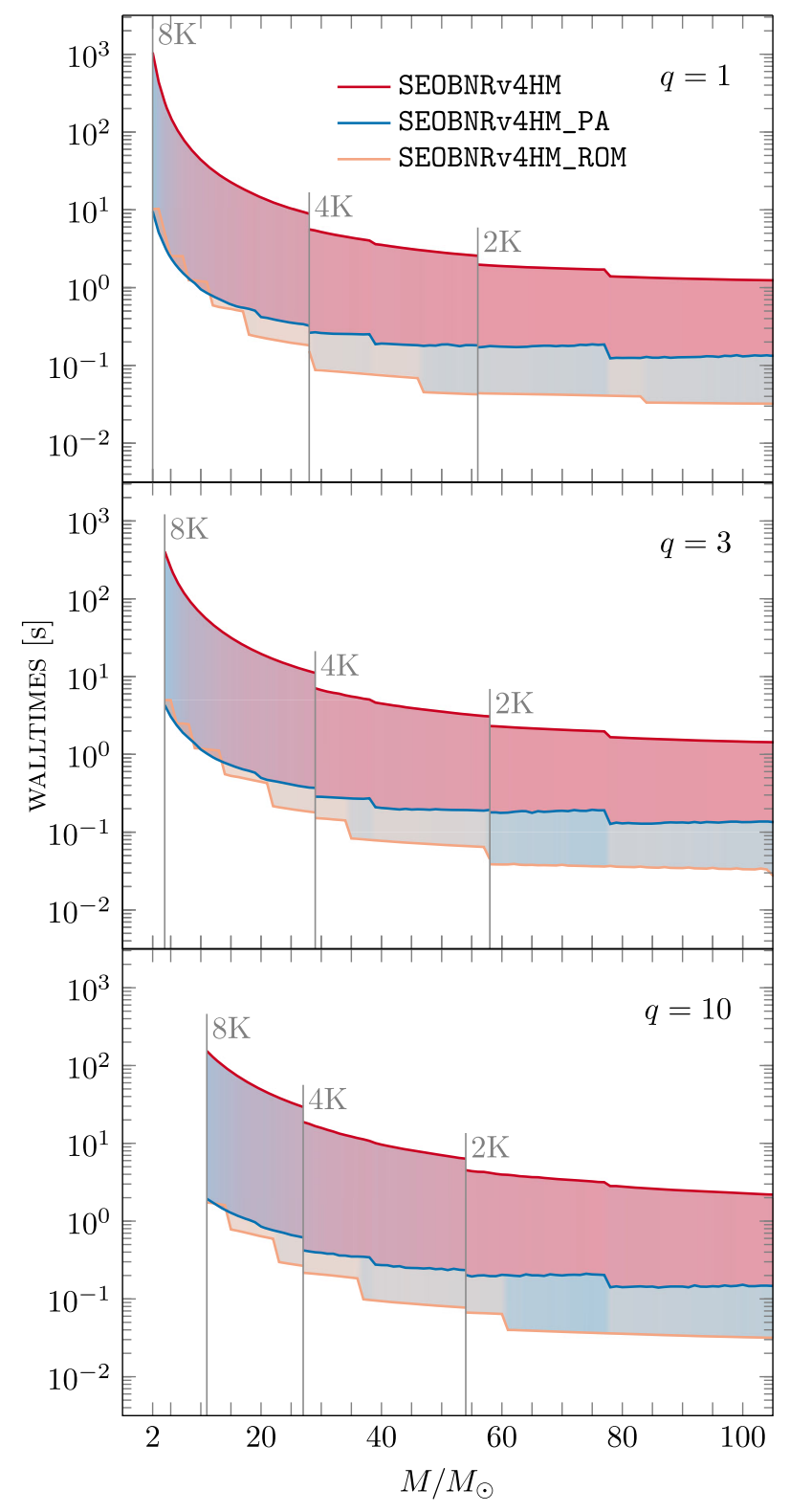

FIG. 1. Benchmark of the SEOBNRV4HM PA waveform model against 2 other well known and commonly used models. Compared to the SEOBNRV4HM model, the post-adiabatic model is between 10 and $10^{2}$ times faster depending on the total mass (for a starting frequency of $10 \mathrm{~Hz}$ ). The frequency-domain SEOBNRV4HM_ROM model is faster for high total mass $M$, but the two models have near-equal performance in the low-totalmass regime. In these tests, the compact objects have spins $\chi_{1}=0.8$ and $\chi_{2}=0.3$, and the sampling rate has been chosen so that it is large enough to resolve the $(5,5)$ mode for large total mass, but also to never exceed $8192 \mathrm{~Hz}$.

available in LALSimulation: against SEOBNRV4HM [45], SEOBNRV4HM_ROM [81] and SEOBNRV4T surrogate [79] in computational efficiency, and against SEOBNRV4HM in accuracy. Figure 1 shows the time for generating a waveform for total masses between 2 and $100 M_{\odot}$, with starting frequency of $10 \mathrm{~Hz}$, and for

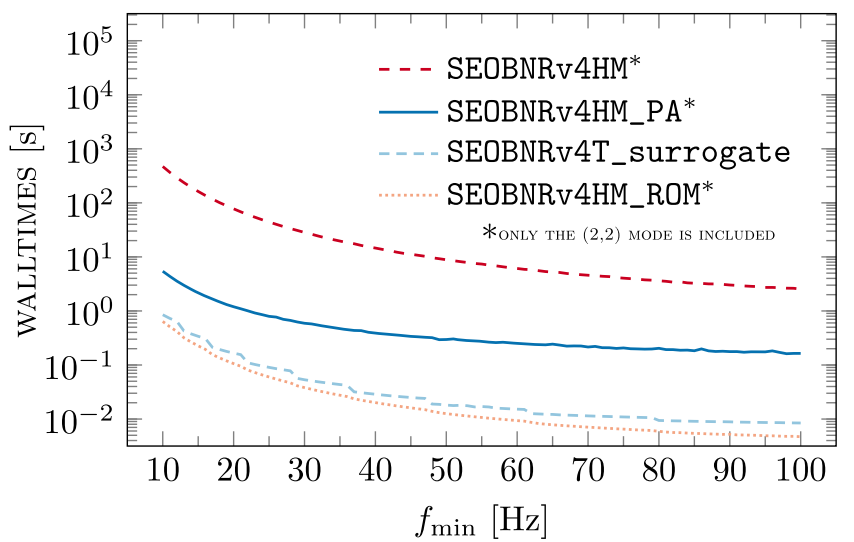

FIG. 2. Benchmark of the SEOBNRV4HM_PA waveform model against SEOBNRV4HM and the surrogate model SEOBNRV4T surrogate for a $1.4 M_{\odot}+1.4 M_{\odot}$ binary with no spins for a range of starting frequencies $f_{\min }$. Since the SEOBNRv4T_ surrogate model only includes the $(\ell,|m|)=(2,2)$ mode, the other models were modified to only compute this mode. All waveforms were sampled at $8192 \mathrm{~Hz}$.

three values of the mass ratio, $q=\{1,3,10\}$. The SEOBNRV4HM_PA model performs significantly faster than the SEOBNRV4HM model across all values of the total mass $M$. The speedup is most significant for lower total mass $(\sim 60 \times)$, and drops to its lowest for high total mass $(\sim 10 \times)$. Most importantly, the speedup is substantial $(\sim 30 \times)$ for $M \sim 40-60 M_{\odot}$, where many of the events of current interest lie. For $M \gtrsim 10 M_{\odot}$, the time to generate a waveform using SEOBNRV4HM_PA is less than $1 \mathrm{~s}$.

Comparing to the frequency-domain SEOBNRv4HM ROM model, we see that, as expected, the reduced order model is faster in almost all cases, except for very low total mass $\left(M \lesssim 10 M_{\odot}\right)$ where the two models are comparable in terms of computational cost (see Fig. 1).

As a further test, the SEOBNRV4HM PA model was benchmarked against the SEOBNRV4 T_surrogate model, which only includes the $(\ell,|m|)=(\overline{2}, 2)$ mode. Figure 2 compares the time for generation of a waveform with the SEOBNRV4HM_PA and SEOBNRV4T_surrogate models where only the quadrupole mode is computed, at different sampling rates, and with different starting frequencies, for a $1.4 M_{\odot}+1.4 M_{\odot}$ BNS with no spins.

Finally, we benchmark the time necessary to generate each higher-oder multipole of the model. Figure 3 shows the times to generate waveforms with the SEOBNRV 4 HM PA model for which all $(\ell,|m|)$ modes with $\ell \leq \ell_{\max }$ (see the legend) are resolved at initial frequency of $f_{0}=20 \mathrm{~Hz}$. As expected, adding higher multipoles increases the Nyquist frequency and the cost for generating the waveform. The figure demonstrates the difference in time that it takes to compute each of the modes of the model. Another test demonstrated that generating additional, higherorder modes at a fixed sampling rate incurs negligible computational cost to the waveform generation. 


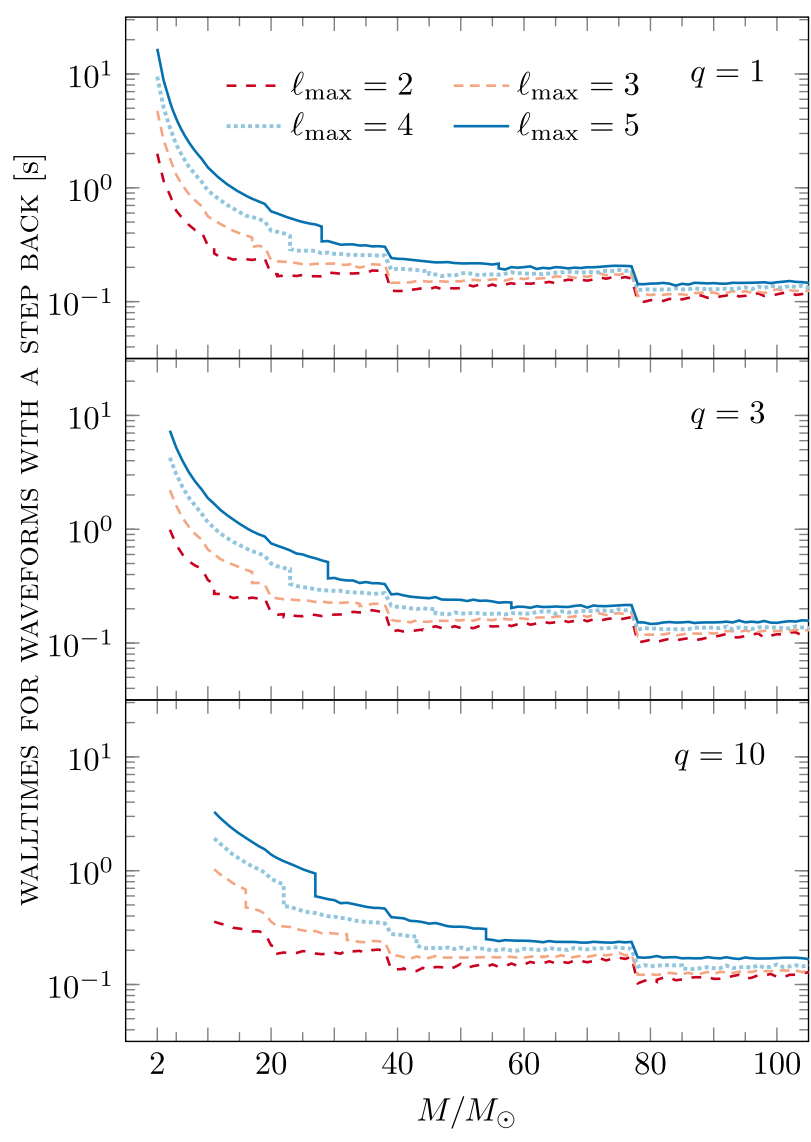

FIG. 3. Benchmark of the SEOBNRV4HM_PA waveform with different highest-order modes allowed. For each mass ratio $q$ and $(\ell, m)$ mode, the initial frequency was modified so that this mode is included at $f=20 \mathrm{~Hz}$. The compact objects have spins $\chi_{1}=0.8$ and $\chi_{2}=0.3$, and the sampling rate has been chosen so that it is large enough to resolve the $(5,5)$ mode for large total mass, but also to never exceed $8192 \mathrm{~Hz}$.

\section{B. Accuracy of the model}

To assess the accuracy of our model with respect to SEOBNRV4HM we use the notion of unfaithfulness as outlined below.

In general, the GW signal from a non-precessing, quasicircular BBH is characterized by a total of 11 parameters: the binary companion masses $m_{1}$ and $m_{2}$, the (dimensionless) component spins $\chi_{1}$ and $\chi_{2}$, which can be aligned or antialigned with the orbital angular momentum, the orientation of the binary in the source frame $\left(l, \varphi_{0}\right)$, the sky location of the source in the detector frame $(\theta, \phi)$, and the luminosity distance $d_{L}$, the time of arrival $t_{c}$, and the polarization angle $\psi$. The detector response can be written as

$$
h(t)=F_{+}(\theta, \phi, \psi) h_{+}\left(\lambda, t_{c} ; t\right)+F_{\times}(\theta, \phi, \psi) h_{\times}\left(\lambda, t_{c} ; t\right),
$$

where $\lambda=\left(m_{1}, m_{2}, \chi_{1}, \chi_{2}, l, \varphi_{0}, d_{L}\right)$ are the binary parameters and the functions $F_{\{+, \times\}}(\theta, \phi, \psi)$ are the antenna patterns (see $[105,106])$. This can be cast as

$h(t)=\mathcal{A}(\theta, \phi)\left(\cos (\kappa) h_{+}\left(\lambda, t_{c} ; t\right)+\sin (\kappa) h_{\times}\left(\lambda, t_{c} ; t\right)\right)$.

Here $\kappa \equiv \kappa(\theta, \phi, \psi)$ is the effective polarization [85], defined as

$$
\exp (i \kappa(\theta, \phi, \psi))=\frac{F_{+}(\theta, \phi, \psi)+i F_{\times}(\theta, \phi, \psi)}{\mathcal{A}(\theta, \phi)},
$$

where the overall amplitude function $\mathcal{A}(\theta, \phi)$ is

$$
\mathcal{A}(\theta, \phi)=\sqrt{F_{+}^{2}(\theta, \phi, \psi)+F_{\times}^{2}(\theta, \phi, \psi)} .
$$

We can now define the match between a GW signal $h_{\mathrm{S}}(t)$ (which for us is SEOBNRV4HM) and a template waveform $h_{\mathrm{T}}(t)$ (which is SEOBNRv4HM_PA) (see [107]):

$$
\mathcal{F}\left(l_{\mathrm{S}}, \varphi_{0 \mathrm{~S}}, \kappa_{\mathrm{S}}\right)=\max _{t_{c}, \varphi_{0 \mathrm{~T}}, \kappa_{\mathrm{T}}}\left[\left.\frac{\left(h_{\mathrm{S}}, h_{\mathrm{T}}\right)}{\sqrt{\left(h_{\mathrm{S}}, h_{\mathrm{S}}\right)\left(h_{\mathrm{T}}, h_{\mathrm{T}}\right)}}\right|_{\substack{m_{1,2 \mathrm{~s}}=m_{1,2 \mathrm{~T}} \\ x_{1,2 \mathrm{~S}}=\chi_{1,2} \\ u_{\mathrm{s}}=l_{\mathrm{T}}}}\right],
$$

where the parameters denoted with $\mathrm{S}(\mathrm{T})$ refer to the signal (template) waveform. For the purposes of this calculation, we marginalize over the phase $\phi_{0 \mathrm{~T}}$, the effective polarization $\kappa_{\mathrm{T}}$, and the time of arrival $t_{c}$, and use the familiar definition for the inner product between two waveforms $[105,106]$ :

$$
(x, y) \equiv 4 \Re\left[\int_{f_{\text {low }}}^{f_{\text {high }}} \mathrm{d} f \frac{\tilde{x}(f) \tilde{y}^{*}(f)}{S_{\mathrm{n}}(f)}\right],
$$

where a $\sim$ denotes a Fourier transform, a $*$ denotes a complex conjugate, and finally $S_{\mathrm{n}}(f)$ is the one-sided power spectral density (PSD) of the detector noise.

Here, we use $f_{\text {low }}=20 \mathrm{~Hz}, f_{\text {high }}=2048 \mathrm{~Hz}$, and the Advanced LIGO "zero-tuned high-power" design sensitivity curve [108]. The definition of the faithfulness in Eq. (3.6) depends on the signal parameters $\left(l_{\mathrm{s}}, \varphi_{0 \mathrm{~s}}, \kappa_{\mathrm{S}}\right)$ and therefore allows us to work with either the maximum or the averaged unfaithfulness (or mismatch) $1-\mathcal{F}\left(l_{\mathrm{s}}, \varphi_{0 \mathrm{~s}}, \kappa_{\mathrm{s}}\right)$ :

$$
\mathcal{U}_{\max } \equiv \max _{\left\{l_{\mathrm{s}}, \varphi_{0 s}, \kappa_{\mathrm{s}}\right\}}[1-\mathcal{F}] \equiv 1-\min _{\left\{l_{\mathrm{s}}, \varphi_{0 \mathrm{~s}}, \kappa_{\mathrm{s}}\right\}}[\mathcal{F}],
$$

$$
\begin{aligned}
\overline{\mathcal{U}} & \equiv\langle 1-\mathcal{F}\rangle_{\left\{l_{\mathrm{s}}, \varphi_{0 \mathrm{~s}}, \kappa_{\mathrm{S}}\right\}} \\
& \equiv 1-\frac{1}{8 \pi^{2}} \int_{0}^{2 \pi} \mathrm{d} \kappa_{\mathrm{S}} \int_{0}^{\pi} \mathrm{d} l_{\mathrm{S}} \sin \left(l_{\mathrm{S}}\right) \int_{0}^{2 \pi} \mathrm{d} \varphi_{0 \mathrm{~s}} \mathcal{F} .
\end{aligned}
$$

Using these definitions of the unfaithfulness, we examine the accuracy of the post-adiabatic (PA) model in 


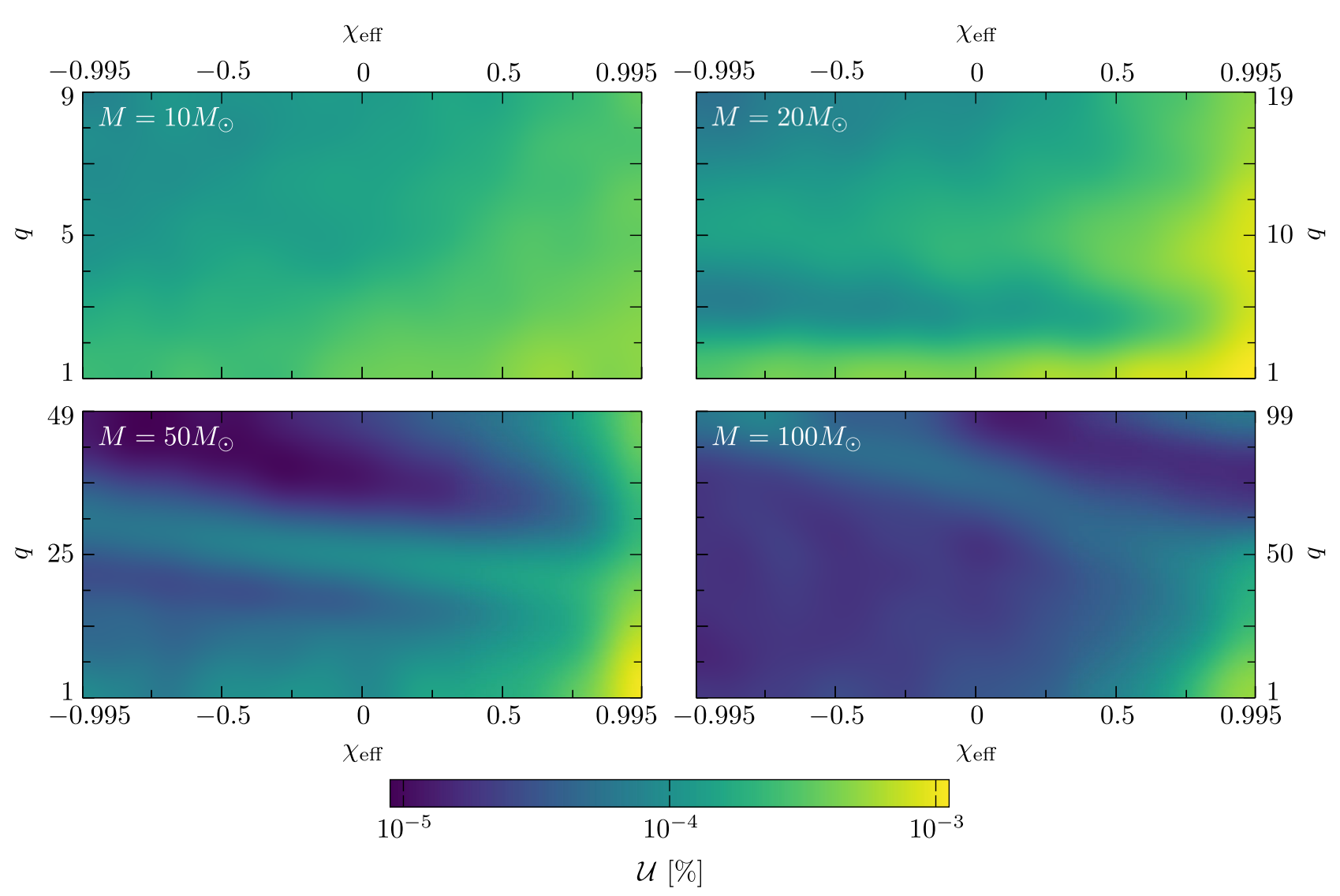

FIG. 4. Unfaithfulness $\overline{\mathcal{U}}$ (in percent) between the SEOBNRV4HM_PA and SEOBNRv4HM models as a function of mass ratio $q$ and effective spin $\chi_{\text {eff }}$ for 4 different values of the total mass $M$.

comparison with SEOBNRv4HM. Figure 4 shows the maximum unfaithfulness $\mathcal{U}_{\max }$ across the parameter space of the effective spin $\chi_{\mathrm{eff}}$ and the mass ratio $q$ for 4 separate values of the total mass $M$. We find that in all cases that were presented, the unfaithfulness is below $\mathcal{O}(0.01 \%)$, which makes our model comparable in accuracy to SEOBNRV4HM_ROM [81]. The variations of the unfaithfulness for a fixed total mass can be explained in terms of the length of the waveform and the heuristic condition which we use to transition from PA inspiral to merger and ringdown.

The variations across the separate panels of Fig. 4 can be better expressed in the form of a histogram of the average unfaithfulness $\overline{\mathcal{U}}$, which is shown in Fig. 5. The distribution for each value of the total mass $M$ peaks at a higher value of the unfaithfulness, which is consistent with the fact that lower-mass binaries undergo a longer coalescence inside the frequency band relevant to LIGO. In all cases, however, the average unfaithfulness stays below $\mathcal{O}(0.01 \%)$.

\section{PARAMETER ESTIMATION STUDY}

While the previous section deals with the absolute performance of the SEOBNRV4HM_PA model, it is important to demonstrate that these benchmarks translate into a faster and reliable parameter estimation (PE) analyses. For this purpose, two PE studies were performed with the SEOBNRV4HM_PA model, as well as some of the established waveform models (SEOBNRV4HM or SEOBNRV4HM $\mathrm{ROM})$. The first one is an injection study involving a synthetic

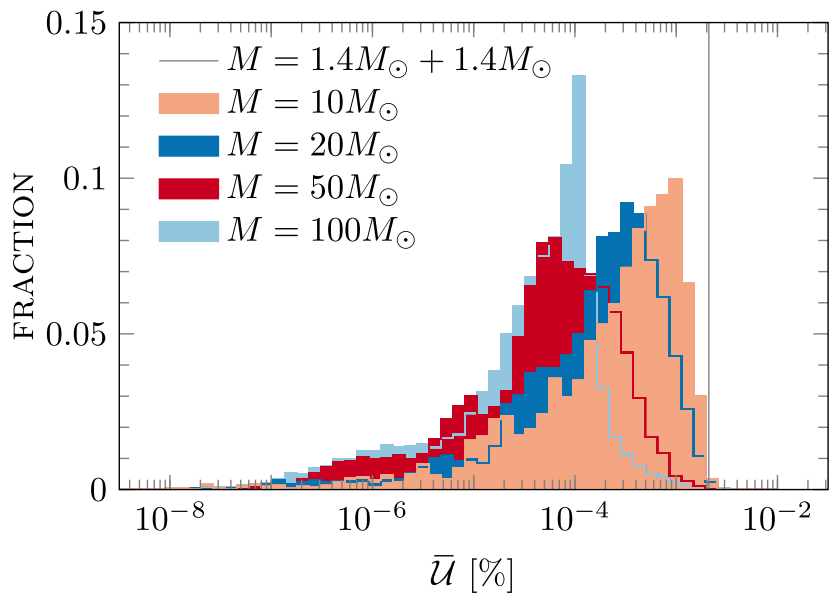

FIG. 5. Histogram of the unfaithfulness $\overline{\mathcal{U}}$ (in percent) between the SEOBNRV4HM_PA and SEOBNRV4HM models for 4 different values of the total mass $M$ and the case of a $1.4 M_{\odot}+1.4 M_{\odot}$ BNS. 


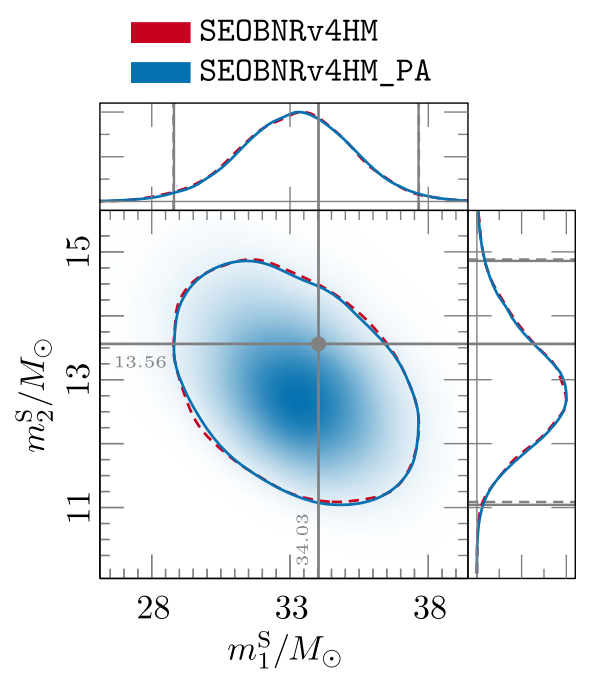

(a)

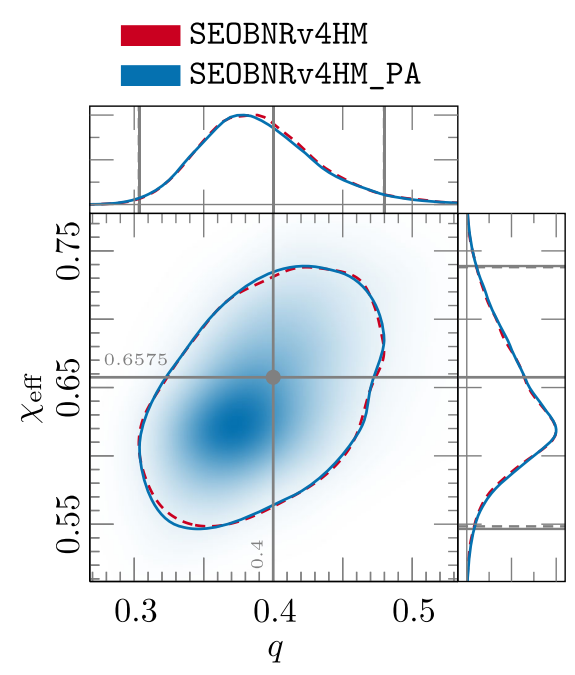

(b)

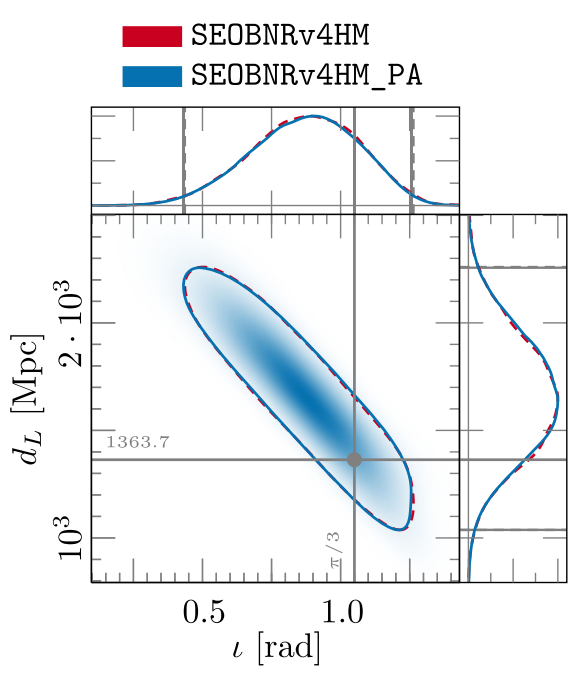

(c)

FIG. 6. Parameter estimation results for the synthetic injection. The 2-dimensional posterior plots show the $90 \%$ confidence regions for the parameters. The grey vertical lines in the 1-dimensional plots show the projections of these confidence regions. The grey labeled lines denote the injection values. Note the excellent agreement between SEOBNRV4HM_PA and SEOBNRV4HM waveform models. (a) Marginalized 2D posterior for the compo- nent source-frame masses $m_{1}$ and $m_{2}$. (b) Marginalized 2D posterior for the mass ratio $q$ and the effective spin $\chi_{\text {eff }}$. (c) Marginalized 2D posterior for the lumi- nosity distance $d_{L}$ and the inclination of the binary $\theta$.

signal. The data was then analyzed using SEOBNRV4HM_PA and SEOBNRV4HM models. The second PE study was performed on data from the event GW190412. It was analyzed using the SEOBNRV4HM PA and the SEOBNRV4HM ROM models.

In both cases, the parameter estimation was done using the Markov-chain Monte Carlo code LALinferenceMCMC [97]. The results of these studies are presented below in the following subsections. We also note that PE studies using the TEOBResums waveform model with the PA approximation were done in Refs $[92,93,95,109,110]$.

\section{A. Injection-based study}

The choice of parameters for this PE study was made so that it could emphasise the speedup which the SEOBNRV4HM_PA model offers, but also to manifest higher-order modes in the signal. The injected signal has total mass $M=60 M_{\odot}$ with mass ratio $1 / q \approx 0.4$, aligned spins $\left(\chi_{1}, \chi_{2}\right)=(0.8,0.3)$, at a distance of approximately $1364 \mathrm{Mpc}$ (corresponding to signal-to-noise ratio (SNR) of $\sim 22$ ) and at an inclination of $\pi / 3$. The injection was made using the SEOBNRV4HM model, while the subsequent analysis was performed using both the SEOBNRV4HM and the SEOBNRV4HM_PA models in order to judge the performance of the post-adiabatic (PA) model.

The results of the parameter estimation analysis can be summarized in the series of marginalized 2-dimensional posterior plots in Fig. 6. Figure 6(a) shows the plot for the component source-frame masses $m_{1}^{s}$ and $m_{2}^{s}$; Fig. 6(b) shows the plot for the mass ratio $q$ and the effective spin $\chi_{\text {eff }}$. Finally, Fig. 6(c) shows the posterior for the luminosity distance $d_{L}$ [cf. Eq. (2.11)] and the inclination angle $l$. The plots demonstrate that the distributions of the posterior samples are in very good agreement between the two models, which demonstrates the reliability of SEOBNRV4HM_PA in PE studies. The Jensen-Shannon (JS) divergence between the 1-dimensional posteriors which are shown in Fig. 6 is always at $\mathcal{O}\left(10^{-3}\right)$ or below, which is consistent with the effects of stochastic sampling on the recovered quantities [111]. Finally, the PE study using the SEOBNRV4HM_PA model had an associated computational cost about 10 times lower than the one performed using the model; this is a due to the speedup presented in Sec. III and further computational overhead associated with the LALinferenceMCMC pipeline.

\section{B. GW190412 parameter estimation}

It is more interesting to demonstrate the viability of the SEOBNRV4HM_PA model on a real GW-event. The GW190412 from the LIGO O3a catalogue is a good candidate for such a study due to its parameters - total mass of $\lesssim 40 M_{\odot}\left(m_{1} \approx 30 M_{\odot}\right.$ and $m_{2} \approx 8 M_{\odot}$, asymmetric mass ratio of about 3 , and dimensionless spin of the massive companion between 0.22 and 0.6 [12,112-114]. The total mass and mass ratio suggest that waveforms for this event would be computationally much more efficient using the SEOBNRV4HM_PA model-which means it is particularly suitable to study this event. The analyses were performed with both the SEOBNRV4HM_PA and SEOBNRV4HM ROM models, since any analysis with the 


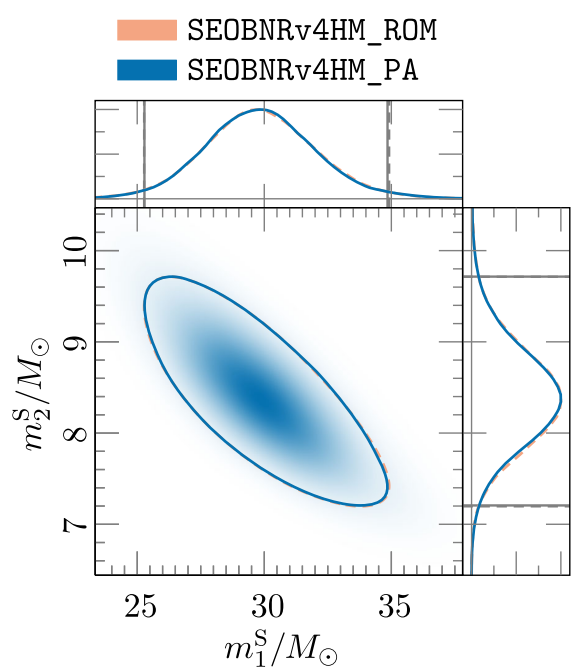

(a)

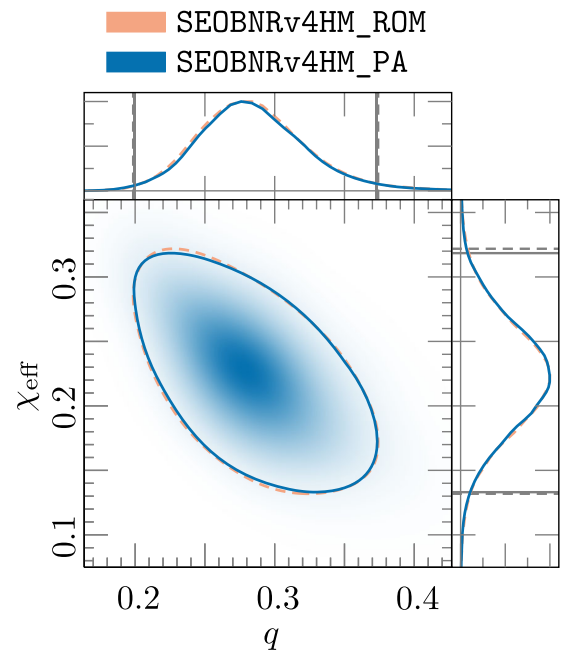

(b)

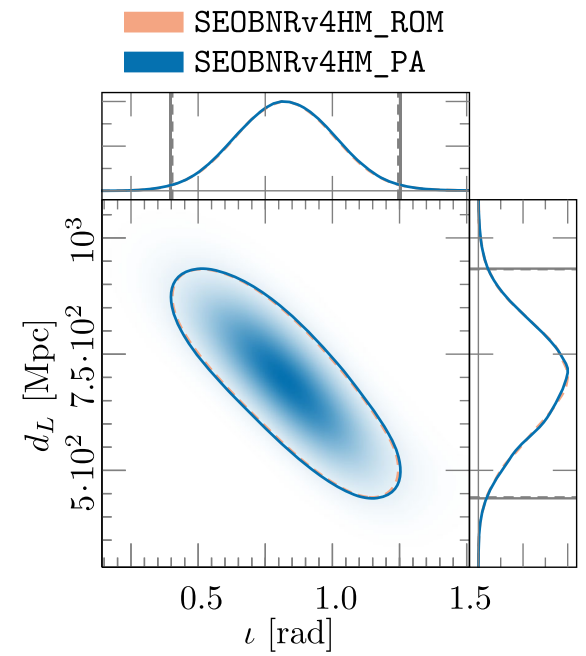

(c)

FIG. 7. Parameter estimation results for the event GW190412. The 2-dimensional posterior plots show the $90 \%$ confidence regions for the parameters. The grey vertical lines in the 1-dimensional plots show the projections of these confidence regions. There is an excellent agreement between the results with the SEOBNRV4HM_PA and the SEOBNRV4HM_ROM models. (a) Marginalized 2D posterior for the component source-frame masses $m_{1}$ and $m_{2}$ (b) Marginalized $2 \mathrm{D}$ posterior $\overline{\text { for }}$ the mass ratio $q$ and the effective spin $\chi_{\text {eff }}$ (c) Marginalized 2D posterior for the luminosity distance $d_{L}$ and the inclination of the binary $l$.

SEOBNRV4HM model would have been impractical in terms of the time required to complete it.

The 2-dimensional marginalized posterior plots are shown in Fig. 7. Figure 7(a) shows the posterior for the component source-frame masses $m_{1}^{\mathrm{s}}$ and $m_{2}^{\mathrm{s}}$ of the binary; Fig. 7(b) shows the plot for the mass ratio $q$ and the effective spin $\chi_{\text {eff }}$; and finally, Fig. 7(c) shows the plot for the luminosity distance $d_{L}$ and the inclination angle $t$. The plots demonstrate the incredibly good agreement between the SEOBNRV4HM_ROM and the SEOBNRV4HM_PA models, which is further confirmed by the JS divergence between the samples, which is below the $\mathcal{O}\left(10^{-3}\right)$ level.

In conclusion for this section, the SEOBNRV4HM_PA model may be reliably used for parameter estimation analyses in place of models like SEOBNRV4HM, with no evident caveats which could hinder the results of such analyses. Furthermore, the average speedup in the generation of samples for the MCMC chains was found to be around 1 order of magnitude. The reason that this is less compared to the speedup demonstrated in Fig. 1 is the fact that for the PE studies, the waveforms are generated using starting frequency $f_{0}=20 \mathrm{~Hz}$.

\section{TESTS OF GENERAL RELATIVITY}

In previous sections we have seen that SEOBNRV4HM_PA is a robust, accurate and fast alternative to SEOBNRv 4 HM and can therefore be used as a drop-in replacement. An interesting further application of this is to use the SEOBNRV4HM_PA model for tests of general relativity, where the SEOBNRV4HM model was previously used as a baseline GR model. In particular, we consider a parametrized black hole $(\mathrm{BH})$ ringdown test that measures the deviations of quasinormal mode emission from predictions of GR. We summarize the test briefly here, see [29,115] for more details. In GR, the no-hair conjecture predicts that the physical properties of a (uncharged) $\mathrm{BH}$ are completely determined by its mass and spin. Consequently, the quasinormal modes (QNM) that describe the gravitational waves emitted by a perturbed $\mathrm{BH}$ are also uniquely determined by its mass and spin. Thus one can check the validity of GR by measuring or constraining any deviations in the complex QNM frequencies. The pSEOBNR ringdown analysis uses a parametrized version of a full inspiral-merger-ringdown GW signal model to measure and constrain the (complex) QNM frequencies. Consequently, unlike other ringdown studies restricted to the post-merger signal [116-119], the pSEOBNR analysis makes use of the entire signal power and does not suffer from the ambiguity of a ringdown start-time definition. In PSEOBNR, one starts with the SEOBNRV4HM model, but then introduces deviations $\delta f_{\operatorname{lm} 0}$ and $\delta \tau_{l m 0}$ (which are treated as free parameters) to the QNM frequency and damping time, so that

$$
\begin{aligned}
f_{l m 0} & =f_{\operatorname{lm} 0}^{\mathrm{GR}}\left(1+\delta f_{l m 0}\right), \\
\tau_{l m 0} & =\tau_{\operatorname{lm} 0}^{\mathrm{GR}}\left(1+\delta \tau_{l m 0}\right),
\end{aligned}
$$

and the ringdown signal is different from the GR prediction. Here, $f_{l m 0}^{\mathrm{GR}}$ and $\tau_{l m 0}^{\mathrm{GR}}$ are computed from final mass and spin as predicted by NR fitting formulas. The goal of the test is to infer the values of the $\left(\delta f_{\operatorname{lm} 0}, \delta \tau_{l m 0}\right)$ by doing full parameter estimation. 


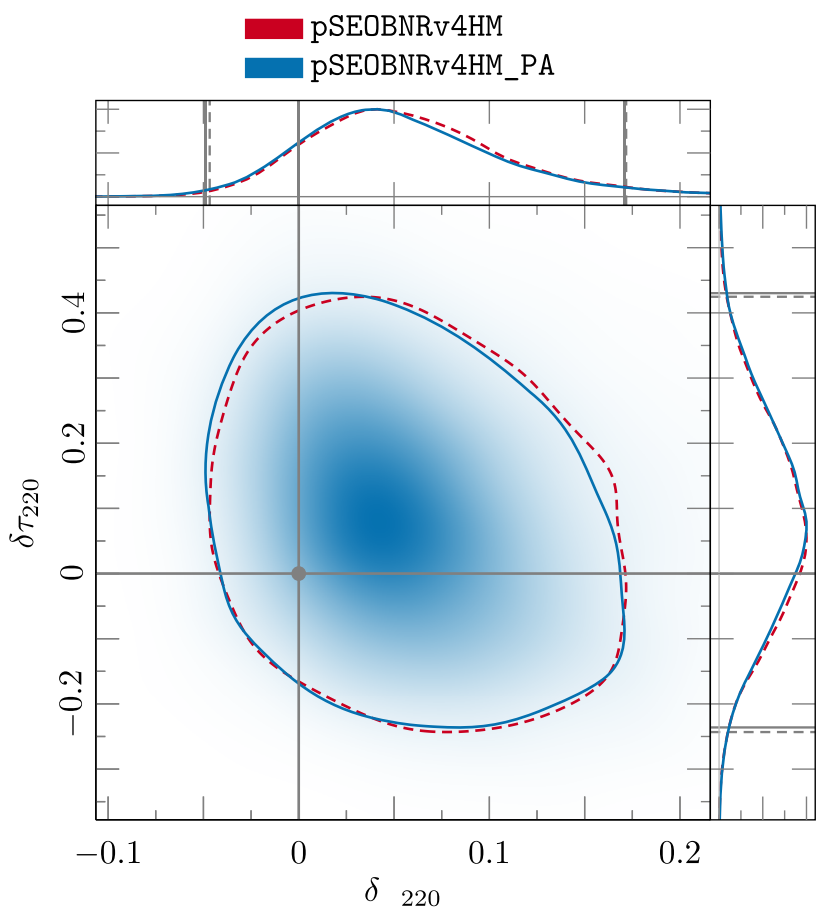

FIG. 8. Posterior distributions for the fractional deviations $\delta f_{220}$ and $\delta \tau_{220}$ in the damping time and frequency as recovered by using SEOBNRV4HM and SEOBNRV4HM_PA. The 2-dimensional posterior plot shows the $90 \%$ confidence regions for the parameters. The grey vertical lines in the 1-dimensional plots show the projections of these confidence regions. The thick grey lines denote the values predicted by GR.

Performing full parameter estimation taking into account deviations from GR is a challenging problem, since it involves sampling a higher-dimensional space (two new parameters for every $(\ell, m)$ mode considered), which means any improvements to waveform speed can lead to even bigger impact on the overall runtime. To check the speed and accuracy of using SEOBNRV4HM_PA for this application, we compare it to the results obtained with SEOBNRV4HM for the first gravitational wave event ever detected, GW150914. We choose this event as it has significant signal-to-noise ratio (SNR) in the merger-ringdown regime. For a high (quasi) equal-mass binary like GW150914, contributions from higher multipoles of the GW signal are expected to be negligible. Hence, we restrict our analysis to just the least-damped dominant QNM, i.e., $\left(f_{220}, \tau_{220}\right)$, keeping the other QNM s fixed at their nominal GR values. In Fig. 8 we show the posterior distributions for the fractional deviations in the damping time and frequency as recovered by using SEOBNRV4HM and SEOBNRV4HM PA. It is evident that the posteriors are in extremely good agreement with the results showing consistency with GR. More quantitatively, the Jensen-Shannon (JS) divergence between the 1-dimensional posteriors is $\mathcal{O}\left(10^{-3}\right)$ for $\delta f_{220}$ and $\delta \tau_{220}$, which is again within the range of what is expected due to stochastic sampling. We find a similarly good level of agreement for the other parameters. Finally, we find a speed-up of $\sim 10$ times when using SEOBNRV4HM_PA instead of SEOBNRV4HM as the base GR model, significantly accelerating inference. With focus shifting from analyses of individual events to population studies, demands on computational resources and personpower are ever-increasing, as demonstrated by large-scale studies in LVK catalog papers [5,7]. Hence, such increases in computational efficiency is immensely important for the future of GW data analysis.

\section{DISCUSSION AND CONCLUSIONS}

We developed the SEOBNRV4HM PA model, which combines the multipolar EOB NR-informed model SEOBNRV4HM with the post-adiabatic (PA) approach for solving the (spin-aligned) binary dynamics (developed and used in the TEOBResums models $[70,71,90,94])$. The resulting model is computationally cheap (at most on the order of seconds and less than 1 second for most of the parameter space) and highly accurate (unfaithfulness less than $\mathcal{O}\left(10^{-3}\right)$ when benchmarked against the SEOBNRV4HM model). Therefore, it can be used as a drop-in replacement for parameter estimation studies and tests of GR for many GW events where the use of the SEOBNRV4HM model would have been impractical.

In Sec. II we presented the EOB formalism and the derivation of the PA equations, together with a discussion on the specifics of transitioning from the PA regime to the merger part of the dynamics. Section III describes the practical implementation of this model in the LALSimulation library of waveform models, and provides the important benchmarks in terms of speed and accuracy of the SEOBNRV4HM_PA model. In particular, we demonstrated that the SEOBNRV4HM_PA model provides a speed-up of 10 to 100 times compared to the SEOBNRV4HM waveform model. Furthermore, we showed that the new model is accurate at a level which allows us to use it for the purposes of LIGO data analysis.

Section IV makes use of the results of the previous section and illustrates the use of the SEOBNRV4HM_PA model in 2 separate parameter estimation studies: recovering an injected synthetic signal and applying the model to analyze the event GW190412. In both cases, we find an extremely good agreement between the results obtained with our model and other established models. Compared to PE with the SEOBNRV4HM model, the study with SEOBNRV4HM_PA completes around 10 times faster. Finally, Sec. $\bar{V}$ depicts the use of the waveform model to a test of GR and discusses the importance of the model for this type of analyses. We find that there is an excellent agreement between the results obtained using the SEOBNRV4HM PA and the established SEOBNRV4HM and SEOBNRV4HM_ROM models.

There are several interesting future directions that can be pursued to apply the PA approximation to other 
SEOBNRV4HM models, notably models that include tidal deformabilities for binary neutron stars [120,121], for which, currently, only the surrogate method has been applied 79]] (see Refs. [91,109] for PA models with tidal effects using TEOBResumS). A more important but challenging extension would involve the precessing SEOBNRV4PHM Hamiltonian [46], which would allow the model to be used more efficiently and for a broader range of GW events when doing parameter-estimation studies. Finally, the PA approximation could also be extended to binary systems on eccentric dynamics. The main challenges with spinprecession and eccentricity is the presence of timescales beyond the orbital and radiation-reaction ones.

\section{ACKNOWLEDGMENTS}

The authors are grateful to Alessandro Nagar and Pierro Rettegno for helpful discussions on the topic of the post-adiabatic approximation, as well as for sharing their post-adiabatic code at the initial stages of this project. The data-analysis studies in this work were obtained with the HPC clusters Hypatia and Minerva at the Max Planck Institute for Gravitational Physics. This research has made use of data, software and/or web tools obtained from the Gravitational Wave Open Science Center (Ref. [122]), a service of LIGO Laboratory, the LIGO Scientific Collaboration and the Virgo Collaboration. LIGO is funded by the U.S. National Science Foundation (NSF). Virgo is funded by the French Centre National de Recherche Scientifique (CNRS), the Italian Istituto Nazionale della Fisica Nucleare (INFN) and the Dutch Nikhef, with contributions by Polish and Hungarian institutes. This paper is based upon work supported by NSF's LIGO Laboratory which is a major facility fully funded by the National Science Foundation.
[1] B. P. Abbott et al. (Virgo and LIGO Scientific Collaborations), Phys. Rev. Lett. 116, 061102 (2016).

[2] B. P. Abbott et al. (LIGO Scientific and Virgo Collaborations), Phys. Rev. X 6, 041015 (2016); 8, 039903(E) (2018).

[3] T. Venumadhav, B. Zackay, J. Roulet, L. Dai, and M. Zaldarriaga, Phys. Rev. D 101, 083030 (2020).

[4] A. H. Nitz, C. Capano, A. B. Nielsen, S. Reyes, R. White, D. A. Brown, and B. Krishnan, Astrophys. J. 872, 195 (2019).

[5] A. H. Nitz, T. Dent, G. S. Davies, S. Kumar, C. D. Capano, I. Harry, S. Mozzon, L. Nuttall, A. Lundgren, and M. Tápai, Astrophys. J. 891, 123 (2020).

[6] B. Zackay, L. Dai, T. Venumadhav, J. Roulet, and M. Zaldarriaga, Phys. Rev. D 104, 063030 (2021).

[7] B. P. Abbott et al. (LIGO Scientific and Virgo Collaborations), Phys. Rev. X 9, 031040 (2019).

[8] R. Abbott et al. (LIGO Scientific and Virgo Collaborations), Phys. Rev. X 11, 021053 (2021).

[9] J. Aasi et al. (LIGO Scientific Collaboration), Classical Quantum Gravity 32, 074001 (2015).

[10] F. Acernese et al. (VIRGO Collaboration), Classical Quantum Gravity 32, 024001 (2015).

[11] Information about gravitational-wave detections made by LIGO to date, https://www.ligo.org/detections.php, accessed: 2021.

[12] R. Abbott et al. (LIGO Scientific and Virgo Collaborations), Phys. Rev. D 102, 043015 (2020).

[13] R. Abbott et al. (LIGO Scientific and Virgo Collaborations), Astrophys. J. Lett. 896, L44 (2020).

[14] R. Abbott et al. (LIGO Scientific and Virgo Collaborations), Phys. Rev. Lett. 125, 101102 (2020).

[15] M. Dominik, E. Berti, R. O'Shaughnessy, I. Mandel, K. Belczynski, C. Fryer, D. E. Holz, T. Bulik, and F. Pannarale, Astrophys. J. 806, 263 (2015).
[16] K. Belczynski, S. Repetto, D. E. Holz, R. O'Shaughnessy, T. Bulik, E. Berti, C. Fryer, and M. Dominik, Astrophys. J. 819, 108 (2016).

[17] B. P. Abbott et al. (KAGRA, LIGO Scientific and Virgo Collaborations), Living Rev. Relativity 23, 3 (2020).

[18] M. Punturo et al., Classical Quantum Gravity 27, 194002 (2010).

[19] D. Reitze et al., Bull. Am. Astron. Soc. 51, 035 (2019), https://baas.aas.org/pub/2020n7i035/release/1.

[20] P. Amaro-Seoane et al. (LISA Collaboration), arXiv:1702 .00786 .

[21] A. Buonanno and B.S. Sathyaprakash, Sources of gravitational waves: Theory and observations, arXiv: 1410.7832 .

[22] B. P. Abbott et al. (LIGO Scientific and Virgo Collaborations), Classical Quantum Gravity 37, 055002 (2020).

[23] I. W. Harry, B. Allen, and B. S. Sathyaprakash, Phys. Rev. D 80, 104014 (2009).

[24] J. Veitch et al., Phys. Rev. D 91, 042003 (2015).

[25] C. Cutler et al., Phys. Rev. Lett. 70, 2984 (1993).

[26] L. Lindblom, B. J. Owen, and D. A. Brown, Phys. Rev. D 78, 124020 (2008).

[27] M. Pürrer and C.-J. Haster, Phys. Rev. Research 2, 023151 (2020).

[28] R. Abbott et al. (LIGO Scientific and Virgo Collaborations), Astrophys. J. Lett. 913, L7 (2021).

[29] R. Abbott et al. (LIGO Scientific and Virgo Collaborations), Phys. Rev. D 103, 122002 (2021).

[30] A. Buonanno and T. Damour, Phys. Rev. D 59, 084006 (1999).

[31] A. Buonanno and T. Damour, Phys. Rev. D 62, 064015 (2000).

[32] T. Damour, P. Jaranowski, and G. Schaefer, Phys. Rev. D 62, 084011 (2000). 
[33] T. Damour, Phys. Rev. D 64, 124013 (2001).

[34] A. Buonanno, Y. Chen, and T. Damour, Phys. Rev. D 74, 104005 (2006).

[35] A. Buonanno, G. B. Cook, and F. Pretorius, Phys. Rev. D 75, 124018 (2007).

[36] T. Damour, B. R. Iyer, and A. Nagar, Phys. Rev. D 79, 064004 (2009).

[37] Y. Pan, A. Buonanno, R. Fujita, E. Racine, and H. Tagoshi, Phys. Rev. D 83, 064003 (2011); 87, 109901(E) (2013).

[38] Y. Pan, A. Buonanno, M. Boyle, L. T. Buchman, L. E. Kidder, H. P. Pfeiffer, and M. A. Scheel, Phys. Rev. D 84, 124052 (2011).

[39] E. Barausse and A. Buonanno, Phys. Rev. D 81, 084024 (2010).

[40] A. Taracchini, Y. Pan, A. Buonanno, E. Barausse, M. Boyle, T. Chu, G. Lovelace, H. P. Pfeiffer, and M. A. Scheel, Phys. Rev. D 86, 024011 (2012).

[41] A. Taracchini et al., Phys. Rev. D 89, 061502 (2014).

[42] Y. Pan, A. Buonanno, A. Taracchini, L. E. Kidder, A. H. Mroué, H. P. Pfeiffer, M. A. Scheel, and B. Szilágyi, Phys. Rev. D 89, 084006 (2014).

[43] A. Bohé, L. Shao, A. Taracchini, A. Buonanno, S. Babak, I. W. Harry, I. Hinder, S. Ossokine, M. Pürrer, V. Raymond et al., Phys. Rev. D 95, 044028 (2017).

[44] S. Babak, A. Taracchini, and A. Buonanno, Phys. Rev. D 95, 024010 (2017).

[45] R. Cotesta, A. Buonanno, A. Bohé, A. Taracchini, I. Hinder, and S. Ossokine, Phys. Rev. D 98, 084028 (2018).

[46] S. Ossokine et al., Phys. Rev. D 102, 044055 (2020).

[47] T. Damour and A. Nagar, Phys. Rev. D 90, 044018 (2014).

[48] A. Nagar, T. Damour, C. Reisswig, and D. Pollney, Phys. Rev. D 93, 044046 (2016).

[49] A. Nagar et al., Phys. Rev. D 98, 104052 (2018).

[50] A. Nagar, A. Bonino, and P. Rettegno, Phys. Rev. D 103, 104021 (2021).

[51] P. Ajith et al., Phys. Rev. Lett. 106, 241101 (2011).

[52] L. Santamaria et al., Phys. Rev. D 82, 064016 (2010).

[53] M. Hannam, P. Schmidt, A. Bohé, L. Haegel, S. Husa, F. Ohme, G. Pratten, and M. Pürrer, Phys. Rev. Lett. 113, 151101 (2014).

[54] S. Husa, S. Khan, M. Hannam, M. Pürrer, F. Ohme, X. J. Forteza, and A. Bohé, Phys. Rev. D 93, 044006 (2016).

[55] S. Khan, S. Husa, M. Hannam, F. Ohme, M. Pürrer, X. J. Forteza, and A. Bohé, Phys. Rev. D 93, 044007 (2016).

[56] L. London, S. Khan, E. Fauchon-Jones, C. García, M. Hannam, S. Husa, X. Jiménez-Forteza, C. Kalaghatgi, F. Ohme, and F. Pannarale, Phys. Rev. Lett. 120, 161102 (2018).

[57] S. Khan, K. Chatziioannou, M. Hannam, and F. Ohme, Phys. Rev. D 100, 024059 (2019).

[58] S. Khan, F. Ohme, K. Chatziioannou, and M. Hannam, Phys. Rev. D 101, 024056 (2020).

[59] G. Pratten, S. Husa, C. Garcia-Quiros, M. Colleoni, A. Ramos-Buades, H. Estelles, and R. Jaume, Phys. Rev. D 102, 064001 (2020).

[60] F. Pretorius, Phys. Rev. Lett. 95, 121101 (2005).

[61] M. Campanelli, C. O. Lousto, P. Marronetti, and Y. Zlochower, Phys. Rev. Lett. 96, 111101 (2006).
[62] J. G. Baker, J. Centrella, D.-I. Choi, M. Koppitz, and J. van Meter, Phys. Rev. Lett. 96, 111102 (2006).

[63] B. Bruegmann, J. A. Gonzalez, M. Hannam, S. Husa, U. Sperhake, and W. Tichy, Phys. Rev. D 77, 024027 (2008).

[64] J. Centrella, J. G. Baker, B. J. Kelly, and J. R. van Meter, Rev. Mod. Phys. 82, 3069 (2010).

[65] A. H. Mroue et al., Phys. Rev. Lett. 111, 241104 (2013).

[66] K. Jani, J. Healy, J. A. Clark, L. London, P. Laguna, and D. Shoemaker, Classical Quantum Gravity 33, 204001 (2016).

[67] J. Healy, C. O. Lousto, Y. Zlochower, and M. Campanelli, Classical Quantum Gravity 34, 224001 (2017).

[68] M. Boyle et al., Classical Quantum Gravity 36, 195006 (2019).

[69] J. Lange, R. O’Shaughnessy, and M. Rizzo, arXiv:1805 .10457.

[70] A. Nagar and P. Rettegno, Phys. Rev. D 99, 021501 (2019).

[71] P. Rettegno, F. Martinetti, A. Nagar, D. Bini, G. Riemenschneider, and T. Damour, Phys. Rev. D 101, 104027 (2020).

[72] S. E. Field, C. R. Galley, J. S. Hesthaven, J. Kaye, and M. Tiglio, Phys. Rev. X 4, 031006 (2014).

[73] M. Pürrer, Classical Quantum Gravity 31, 195010 (2014).

[74] J. Blackman, S. E. Field, C. R. Galley, B. Szilágyi, M. A. Scheel, M. Tiglio, and D. A. Hemberger, Phys. Rev. Lett. 115, 121102 (2015).

[75] M. Pürrer, Phys. Rev. D 93, 064041 (2016).

[76] J. Blackman, S. E. Field, M. A. Scheel, C. R. Galley, D. A. Hemberger, P. Schmidt, and R. Smith, Phys. Rev. D 95, 104023 (2017).

[77] Z. Doctor, B. Farr, D. E. Holz, and M. Pürrer, Phys. Rev. D 96, 123011 (2017).

[78] J. Blackman, S. E. Field, M. A. Scheel, C. R. Galley, C. D. Ott, M. Boyle, L. E. Kidder, H. P. Pfeiffer, and B. Szilágyi, Phys. Rev. D 96, 024058 (2017).

[79] B. D. Lackey, M. Pürrer, A. Taracchini, and S. Marsat, Phys. Rev. D 100, 024002 (2019).

[80] Y. Setyawati, M. Pürrer, and F. Ohme, Classical Quantum Gravity 37, 075012 (2020).

[81] R. Cotesta, S. Marsat, and M. Pürrer, Phys. Rev. D 101, 124040 (2020).

[82] V. Varma, S. E. Field, M. A. Scheel, J. Blackman, L. E. Kidder, and H. P. Pfeiffer, Phys. Rev. D 99, 064045 (2019).

[83] V. Varma, S. E. Field, M. A. Scheel, J. Blackman, D. Gerosa, L. C. Stein, L. E. Kidder, and H. P. Pfeiffer, Phys. Rev. Research 1, 033015 (2019).

[84] D. A. Brown, P. Kumar, and A. H. Nitz, Phys. Rev. D 87, 082004 (2013).

[85] C. Capano, Y. Pan, and A. Buonanno, Phys. Rev. D 89, 102003 (2014).

[86] V. Varma, P. Ajith, S. Husa, J. C. Bustillo, M. Hannam, and M. Pürrer, Phys. Rev. D 90, 124004 (2014).

[87] V. Varma and P. Ajith, Phys. Rev. D 96, 124024 (2017).

[88] I. Harry, J. C. Bustillo, and A. Nitz, Phys. Rev. D 97, 023004 (2018).

[89] C. Kalaghatgi, M. Hannam, and V. Raymond, Phys. Rev. D 101, 103004 (2020).

[90] T. Damour, A. Nagar, and S. Bernuzzi, Phys. Rev. D 87, 084035 (2013). 
[91] A. Nagar, F. Messina, P. Rettegno, D. Bini, T. Damour, A. Geralico, S. Akcay, and S. Bernuzzi, Phys. Rev. D 99, 044007 (2019).

[92] R. Gamba, M. Breschi, S. Bernuzzi, M. Agathos, and A. Nagar, Phys. Rev. D 103, 124015 (2021).

[93] R. Gamba, S. Bernuzzi, and A. Nagar, Phys. Rev. D 104, 084058 (2021).

[94] G. Riemenschneider, P. Rettegno, M. Breschi, A. Albertini, R. Gamba, S. Bernuzzi, and A. Nagar, Phys. Rev. D 104, 104045 (2021).

[95] M. Breschi, R. Gamba, and S. Bernuzzi, Phys. Rev. D 104, 042001 (2021).

[96] T. Damour and A. Nagar, Phys. Rev. D 77, 024043 (2008).

[97] LIGO Scientific and LIGO Scientific Collaborations, LIGO Algorithm Library-LALSuite, free software (GPL) (2018).

[98] A. Burden, R. Burden, and J. Faires, Numerical Analysis, 10th ed. (Cengage, Boston, MA, USA, 2016).

[99] M. Abramowitz and I. Stegun, Handbook of Mathematical Functions with Formulas, Graphs, and Mathematical Tables (1964), Vol. 59, p. 1324.

[100] B. Fornberg, Math. Comput. 51, 699 (1988).

[101] M. H. Holmes, Am. Math. Mon. 121, 723 (2014).

[102] W. H. Press, S. A. Teukolsky, W. T. Vetterling, and B. P. Flannery, Numerical Recipes 3rd Edition: The Art of Scientific Computing, 3rd ed. (Cambridge University Press, Cambridge, 2007).

[103] C. Devine, Z. B. Etienne, and S. T. McWilliams, Classical Quantum Gravity 33, 125025 (2016).

[104] T. D. Knowles, C. Devine, D. A. Buch, S. A. Bilgili, T. R. Adams, Z. B. Etienne, and S. T. Mcwilliams, Classical Quantum Gravity 35, 155003 (2018).

[105] B. S. Sathyaprakash and S. V. Dhurandhar, Phys. Rev. D 44, 3819 (1991).
[106] L. S. Finn and D. F. Chernoff, Phys. Rev. D 47, 2198 (1993).

[107] I. Harry, S. Privitera, A. Bohé, and A. Buonanno, Phys. Rev. D 94, 024012 (2016).

[108] L. Barsotti, P. Fritschel, M. Evans, and S. Gras (LIGO Collaboration), L. Barsotti, Updated Advanced LIGO sensitivity design curve, LIGO Document T1800044-v5 (2018).

[109] S. Akcay, S. Bernuzzi, F. Messina, A. Nagar, N. Ortiz, and P. Rettegno, Phys. Rev. D 99, 044051 (2019).

[110] S. Akcay, R. Gamba, and S. Bernuzzi, Phys. Rev. D 103, 024014 (2021).

[111] I. M. Romero-Shaw et al., Mon. Not. R. Astron. Soc. 499, 3295 (2020).

[112] M. Zevin, C. P. L. Berry, S. Coughlin, K. Chatziioannou, and S. Vitale, Astrophys. J. Lett. 899, L17 (2020).

[113] T. Islam, S. E. Field, C.-J. Haster, and R. Smith, Phys. Rev. D 103, 104027 (2021).

[114] M. Colleoni, M. Mateu-Lucena, H. Estellés, C. GarcíaQuirós, D. Keitel, G. Pratten, A. Ramos-Buades, and S. Husa, Phys. Rev. D 103, 024029 (2021).

[115] A. Ghosh, R. Brito, and A. Buonanno, Phys. Rev. D 103, 124041 (2021).

[116] M. Isi, M. Giesler, W. M. Farr, M. A. Scheel, and S. A. Teukolsky, Phys. Rev. Lett. 123, 111102 (2019).

[117] M. Giesler, M. Isi, M. A. Scheel, and S. Teukolsky, Phys. Rev. X 9, 041060 (2019).

[118] G. Carullo, G. Riemenschneider, K. W. Tsang, A. Nagar, and W. Del Pozzo, Classical Quantum Gravity 36, 105009 (2019).

[119] G. Carullo, W. Del Pozzo, and J. Veitch, Phys. Rev. D 99, 123029 (2019); 100, 089903(E) (2019).

[120] T. Hinderer et al., Phys. Rev. Lett. 116, 181101 (2016).

[121] J. Steinhoff, T. Hinderer, A. Buonanno, and A. Taracchini, Phys. Rev. D 94, 104028 (2016).

[122] https://www.gw-openscience.org. 